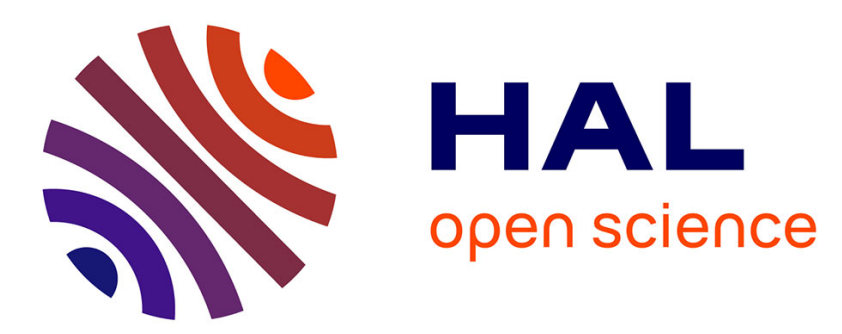

\title{
Simulation of atmospheric secondary hadron and lepton flux from satellite to underground experiments
}

M. Buénerd

\section{To cite this version:}

M. Buénerd. Simulation of atmospheric secondary hadron and lepton flux from satellite to underground experiments. 2nd International Workshop on Matter, Anti-Matter and Dark Matter, Nov 2001, Trento, Italy. pp.1665-1674, 10.1142/S0217751X02011163 . in2p3-00011701

\section{HAL Id: in2p3-00011701 https://hal.in2p3.fr/in2p3-00011701}

Submitted on 22 Feb 2002

HAL is a multi-disciplinary open access archive for the deposit and dissemination of scientific research documents, whether they are published or not. The documents may come from teaching and research institutions in France or abroad, or from public or private research centers.
L'archive ouverte pluridisciplinaire HAL, est destinée au dépôt et à la diffusion de documents scientifiques de niveau recherche, publiés ou non, émanant des établissements d'enseignement et de recherche français ou étrangers, des laboratoires publics ou privés. 


\title{
SIMULATION OF ATMOSPHERIC SECONDARY HADRON AND LEPTON FLUX FROM SATELLITE TO UNDERGROUND EXPERIMENTS
}

\author{
M. BUÉNERD \\ Institut des Sciences Nucléaires, IN2P3/CNRS, \\ 53 av. des Martyrs, 38026 Grenoble cedex, France \\ Received (Day Month Year) \\ Revised (Day Month Year)
}

\begin{abstract}
The successful simulation results obtained in the interpretation of the recent AMS measurements of proton, leptons and light nuclei particle flux, are reviewed. A similar success is being met in the analysis of secondary particle flux measured in the atmosphere (antiprotons, muons) and in underground experiments (neutrinos).
\end{abstract}

Keywords: Cosmic Rays; Atmospheric secondaries; Hadroproduction.

\section{INTRODUCTION}

Recent experiments have reported new measurements of atmospheric secondary particle flux over a broad range altitudes, depending on the particular species, extending from satellite and balloon altitudes, down to ground and underground experiments. This presentation reviews the results obtained recently in a simulation work undertaken to account for the measured flux of secondary particles in terms of dynamical interactions between Cosmic Rays (CR) and the atmosphere.

The primary purpose of the work was to understand the proton, $e^{ \pm}$, and light ion flux measured recently by the AMS experiment ${ }^{1,2,3,4}$. However, while this undertaking was considered, it was also felt that it could contribute fruitfully to various other experiments involving directly or undirectly CR-atmosphere interactions like $\bar{p}$, and lepton flux measurements in balloon experiments, and the underground neutrino experiments, as well as to the evaluation of the secondary particle populations for the preparation of future satellite experiments like AMS2, PAMELA, etc...

The results presented in the following have been reported in scientific journals $5,6,7$, or conference proceedings $8,9,10,11$, while some others are still preliminary and will be reported in forthcoming publications ${ }^{13,14}$.

The first part of the talk will briefly describe the general features of the simulation program. In the second part, the results obtained in the interpretation of the AMS data on the $p, e^{ \pm}, d$ and ${ }^{3} H e$, flux will be reviewed. The third part will be devoted to the simulation of the secondary $\bar{p}$ and $\mu$ flux in the atmosphere, and to 
the atmospheric neutrino flux in underground experiments.

\section{The simulation program}

The basic assumptions on the physics processes governing the production and the propagation of the flux of secondary charged particles in the terrestrial environment, incorporated in the simulation program were the following: - The secondary particles are produced by the interaction between the Cosmic Ray flux (CR) and the atmosphere.

- For the present purpose, the main CR flux can be limited to a good approximation to the Proton and Helium particles. These flux are described in accordance with the recent AMS measurements ${ }^{1,2,4}$ with appropriate corrections due to the solar modulation effects, made.

- The density of atmospheric nuclei can be described according to the currently available model of atmosphere.

- All charged particles propagate in the earth magnetic field described by a multipole development on the geomagnetic angular coordinates.

- For each secondary particle species, particles are generated according to the energy dependent differential cross section for inclusive production, parametrized as described below.

- Secondary particle propagation and interactions are processed the same way as for incoming CRs, leading to the development of atmospheric cascades involving up to about 10 generations of successive collisions. All charged particles undergo energy loss by ionization.

This approach therefore takes into account the particle productions in secondary interactions at all stages inside the atmospheric cascade. This has quite significant effects as it will be seen below.

(For technical and bibliographical details on the above, see ${ }^{5,6}$ ).

\subsection{Production cross section for secondary particles}

With the incoming flux of proton and helium as a common feature to all calculations, the inclusive triple differential cross sections appropriate for each production channel had to be evaluated separately in the event generator. The following production cross sections were used for the calculations of the various secondary particle flux calculated:

\subsubsection{Protons}

Protons produced from the $C R+A \rightarrow p+X$ reactions, with $C R, A$, and $X$ standing for $\mathrm{CR}$ particles, atmospheric nuclei (A weighted average value of the mass), and recoil system, respectively ${ }^{5}$. The calculated cross section included two components corresponding to quasi free (forward) and deep inelastic (backward) scattering processes ${ }^{18}$, respectively. 


\subsubsection{Light nuclei}

Light nuclei were assumed to originate from the $C R+A \rightarrow a+X$ with $a={ }^{2,3} H$, ${ }^{3,4} \mathrm{He}$. Two models were considered and implemented to describe this production, corresponding to different final state kinematics: The fragmentation model and the coalescence model, both in their simplest versions ${ }^{7}$.

\subsubsection{Leptons}

This is a main aspect of the work since several lepton flux have been measured, in balloon, satellite, and underground experiments, which all originate from the same reaction type, at least for the dominant mechanism. The flux calculations for these species are then most tightly and thus sensitively constrained by the data. The main mechanism of lepton generation considered was through the pion production reaction chain $C R+A \rightarrow \pi+X, \pi \rightarrow \mu+\nu, \mu \rightarrow e+\nu+\bar{\nu}$, with $\mathrm{CR}$ standing for Cosmic Ray $\left(p,{ }^{4} \mathrm{He}\right)$, and A for nucleus. The kaon contribution is typically one order of magnitude smaller; It was considered only for the neutrino and muon flux calculations ${ }^{14}$. These cross sections are governing the populations of $e^{ \pm}, \mu^{ \pm}, \nu$ produced in the atmosphere, and it is one of the major successes of the works presented here, to reproduce simultaneously the measured flux of $e^{ \pm}$at the (satellite) altitude of AMS (see also ${ }^{9}$ for the $e^{ \pm}$flux in the atmosphere), of $\mu^{ \pm}$in the atmosphere from ground level to TOA, and the Est-West neutrino asymmetry measured by superK, to a fair accuracy with no adjustable parameter. The inclusive pion differential cross section used in the calculations was based on a modified version of the functional form proposed in ${ }^{16}$, and fitted to a broad range of pion data ${ }^{17}$. Good fits to the data could be obtained through the covered momentum range $(\approx 1.5-450 \mathrm{GeV} / \mathrm{c})$. For the Kaon production, the form and parameters from 16 were used. For the electron flux, the (tertiary) pair production cross section $\gamma+A \rightarrow e^{+} e^{-}+A$ induced by Bremstrahlung photons and $\pi^{0}$ decay photons, was also calculated ${ }^{6}$.

\subsubsection{Antiprotons}

For the antiprotons, a modified form based on the Ref ${ }^{16}$ parametrized functional was used for the description of the inclusive production reaction $C R+A \rightarrow \bar{p}+X$. It was fitted on a set of antiproton data ${ }^{12}$ measured at incident energies between about 12 and $24 \mathrm{GeV}$, for which good fits could be obtained. The work is being extended to higher energies with good results up to $300 \mathrm{GeV}$ in laboratory.

\section{Interpretation of the AMS results}

\subsection{Protons}

The first interpretation effort of the AMS data using the present approach, was devoted to the strong component of protons ("second spectrum") below the ge- 

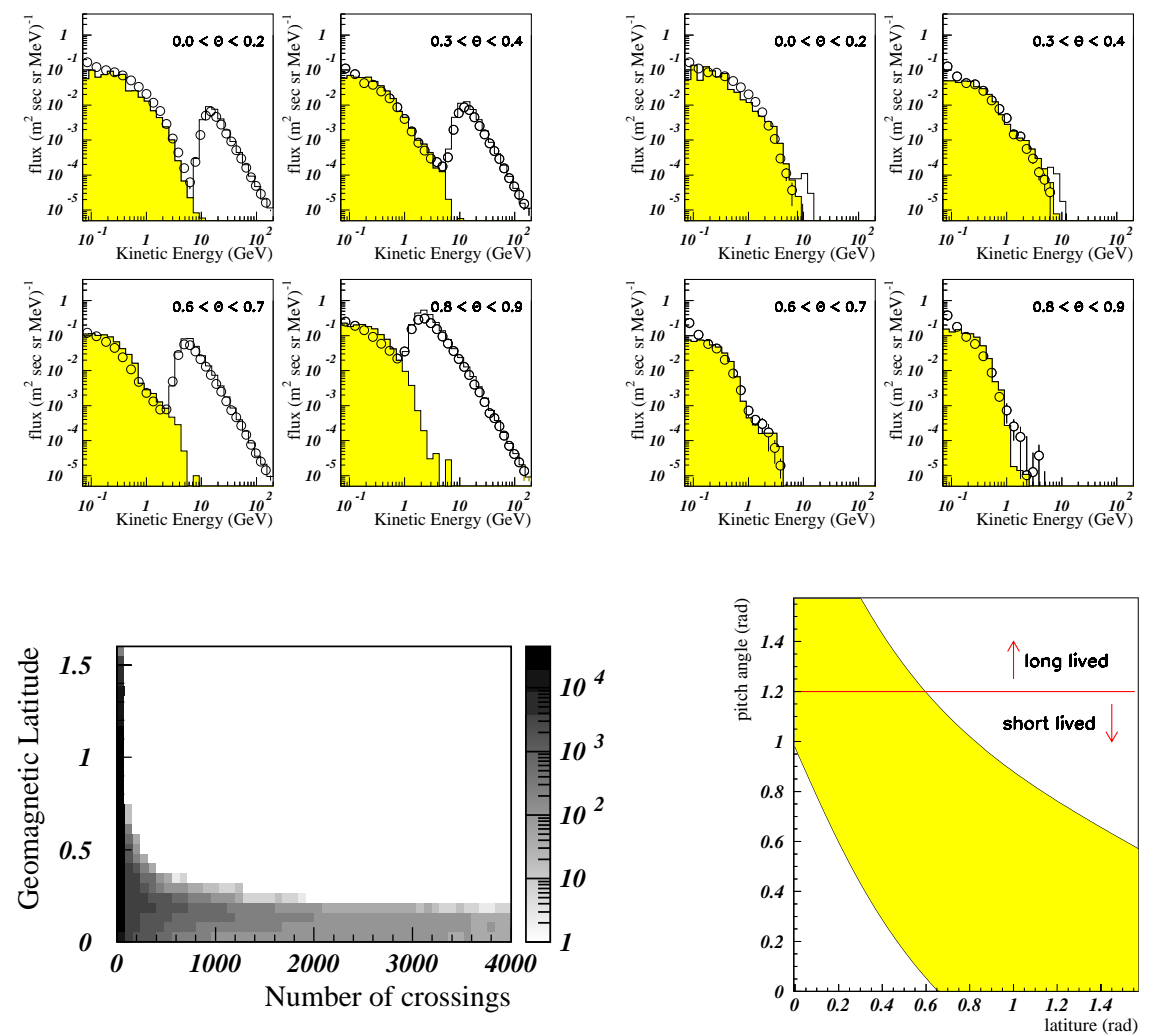

Fig. 1. Top: Experimental upward and downward proton flux measured by AMS compared with the simulation results. Bottom left: Simulated flux of trapped protons. Bottom right: AMS pitch angle acceptance versus latitude, explaining the confinement of the trapped protons in the equator region seen on the left. From Refs. $[5,15]$

omagnetic cutoff $(\mathrm{GC})^{1}$. The results are illustrated on fig 1 . The data are very well reproduced by the calculations for all latitudes, including the strong flux enhancement in the $\mathrm{GeV}$ region of the spectrum for equatorial latitude. The latter appears to be due to trapped or semi-trapped (long lived) particles. Bottom left Fig. 1 shows the scatter plot of the long lived population of the simulated sample, which appears to be confined in the equatorial region. The explanation for this is given on the right hand panel ${ }^{15}$ which shows the particle pitch angle (PA) at the AMS location versus geomagnetic latitude plane. In this plane, trapped particles are allowed only in the area above the horizontal line, i.e. for $\mathrm{PA}>\approx 1.2$, where mirror points of particle trajectories lie outside (above) the atmosphere. Below this line, they are inside the atmosphere, and thus particles cannot be trapped. Particle directions have also to be within the AMS acceptance shown by the broad grey 
stripe going from the upper left to the lower right across the figure. The area of the plane allowed to trapped particles is thus limited to the upper left corner (large pitch angles and low latitudes) limited by the mirror point condition and the limits of the AMS acceptance. The upper limit in latitude $(\approx 0.6 \mathrm{rad})$ is seen to be in agreement with the latitude distribution of the simulated population of long lived protons (left panel).

\subsection{Electrons and positrons}

The flux of $e^{+}$and $e^{-}$particles measured by AMS was surprising by several respects: First, it displayed a similar strong second spectrum below GC as observed for protons; Second, The $e^{+}$over $e^{-}$flux ratio evolved from about four around the equator down to about one in the polar region ${ }^{3}$. These results are quite well reproduced by the calculations, as seen on fig 2 . The analysis of the simulation data showed that the strong charge asymmetry observed for equatorial latitudes is due to a combined effect of the East-West (EW) dependence of the GC, with the forward peaking of the lepton production reactions, and the absorption effect of the
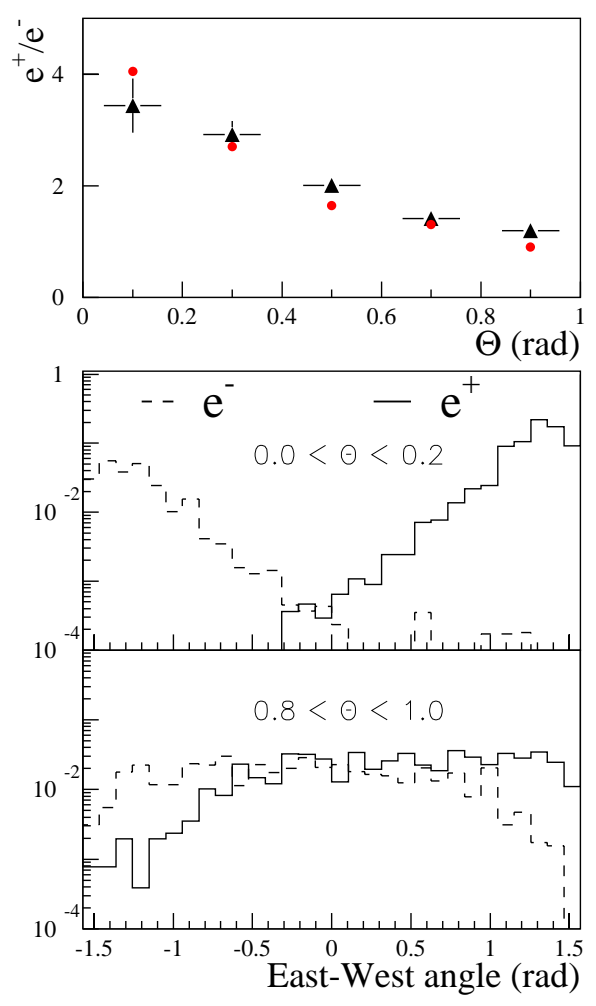

Fig. 2. Top: Experimental (full circles) and simulated (triangle) lepton asymmetry versus geomagnetic latitude. Middle: Eastbound and Westbound lepton spectra showing the large EW asymmetry for equatorial latitudes. Bottom: Same for polar region showing as expected, a vanishing asymmetry. From Ref.[6] 
atmosphere ${ }^{6}$.

\subsection{Light ions}

The AMS data surprisingly uncovered the existence of a small but sizeable subGC component of ${ }^{3} \mathrm{He}$, whereas no ${ }^{4} \mathrm{He}$ flux could be detected (above some upper limits set by the experimental conditons), while the latter is dominating the former in the CR flux by a sound order of magnitude ${ }^{4}$. This apparent paradox found a natural explanation in the framework of the nuclear coalescence model which allowed to account quantitatively for all these features with a good accuracy ${ }^{7}$. This is illustrated on fig 3 left, where the experimental energy spectrum and latitude distribution of ${ }^{3} \mathrm{He}$ particles are seen to be well reproduced by the calculations. This deuterium flux below GC is also very well reproduced by calculations using the same model (fig 3 right). This consistent agreement between data and calculations (no adjustable parameter in the calculations), for two populations of light nuclei provides a strong support to the validity of the theoretical basis of the approach.
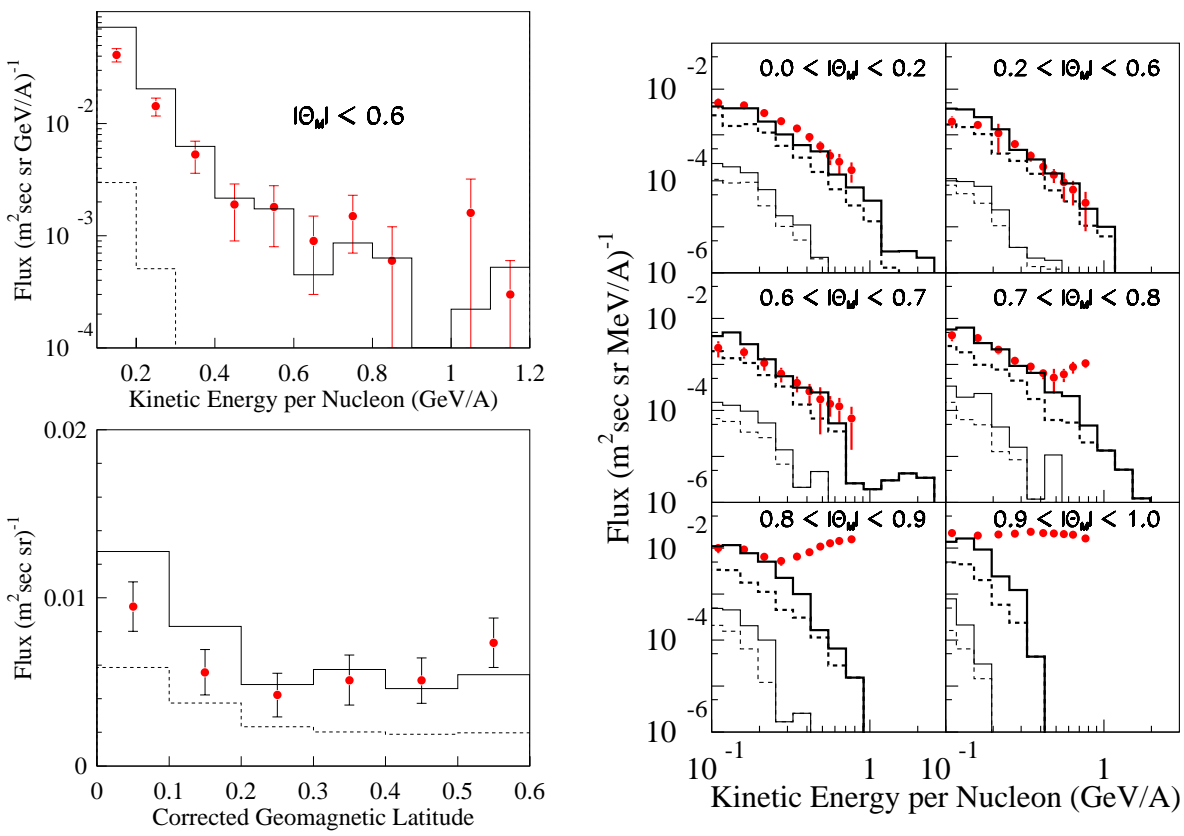

Fig. 3. Left top: Experimental (full circles) and simulated (histograms) ${ }^{3} \mathrm{He}$ flux distributions below GC, versus energy (integrated over latitude). Left bottom: Same as above versus latitude (integrated over energy). Right: Deuterium $\left({ }^{2} \mathrm{H}\right)$ flux measured by AMS (full circles) and subGC component calculated by simulation (histogram), simultaneously with the ${ }^{3} \mathrm{He}$ flux. The lower histograms give the calculated tritium $\left({ }^{3} \mathrm{H}\right)$ flux. In all panels dashed histograms show the $\mathrm{CR}$ $H e$ induced contributions. From Ref.[7] 


\section{Secondary atmospheric particle flux}

The successful interpretation of the AMS data opened new prospects for calculating the flux of secondary atmospheric particles like antiprotons, muons, and neutrinos relevant to several ongoing experimental programs, and to future new experiments.

\subsection{Antiprotons}

The BESS ${ }^{19}$ and CAPRICE ${ }^{21}$ experiments are studying the antiproton CR flux in high altitude balloon experiments. In these experiments, the raw data have to be corrected for the atmospheric $\bar{p}$ production to obtain the galactic flux. Fig. 4 shows the CR $\bar{p}$ flux obtained by BESS, together with the applied corrections (curve), and those calculated in the present work (histogram). The flux expected from the simulation results apppears to be significantly larger than the values obtained from the transport equation approach. This is observed for both the BESS (left) and CAPRICE (middle) data. Fig 4 right shows the preliminary results of measurements at terrestrial altitude $(2770 \mathrm{~m})$ from BESS ${ }^{20}$ compared to the preliminary simulation results at this altitude ${ }^{13}$. The agreement is excellent and consistent with the previously quoted results, showing that the particle production in the atmosphere seems to be fairly well understood and the underlying dynamics and kinematics appropriately treated. It must be pointed out however that the calculated proton flux at this altitude significantly overestimate the data from the same experiment, a point of disagreement currently under examination. At sea level, the antiproton flux in the GeV range is calculated to be of the order of $2\left[\mathrm{~m}^{2} / \mathrm{sr} / \mathrm{GeV} / \mathrm{c} / \mathrm{mn}\right]^{-1}$. A small flux, but large enough to allow the testing of the $\bar{p}$ identification capability of space detectors on the ground ${ }^{11,13}$.

The antineutron flux at terrestrial altitudes is being calculated in the same
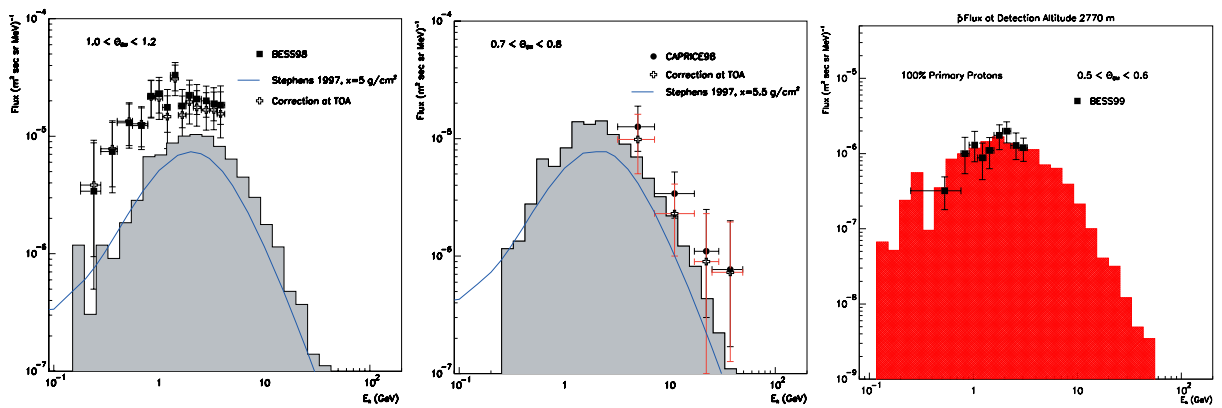

Fig. 4. Experimental $\bar{p}$ flux (symbols) measured in the atmosphere. Left: Low energy BESS measurements at high altitude; Middle: high energy CAPRICE data; Right: $2770 \mathrm{~m}$ BESS measurements (preliminary [20]). The data are compared to (preliminary) simulation results (histograms). The curves show the calculated corrections (atmospheric flux) to the raw flux in the original works. 
conditions in the perspective of an experimental project ${ }^{22}$.

\subsection{Muons}

The muon flux measurements together with the $e^{ \pm}$measurements constitute the most constraining body of data currently available for a stringent test of any theoretical or phenomenological approach of the atmospheric production of leptons. This is of major importance regarding the atmospheric neutrino issue which interpretation relies to some extent on calculations ${ }^{25}$ and of which some results are still awaiting for a quantitative interpretation (see below) ${ }^{14}$. The subject has been addressed in several previous works using various theoretical approaches (see ${ }^{14}$ for the context).

Fig 5 shows a set of $\mu^{-}$spectra measured recently by the CAPRICE ${ }^{23}$ and HEAT ${ }^{24}$ experiments for altitudes ranging from ground level up to $38 \mathrm{~km}$, compared with the simulations results ${ }^{14}$. The right panel shows the ratio of calculated to measured values. The agreement between calculation and data is very good through the range of altitudes, but for the $38 \mathrm{~km}$ spectrum for which the calculations significantly underestimate the experimental flux for low energy muons below about $1 \mathrm{GeV}$. This defect seems to be related to the low energy pion yield of the event generator. It has turned out that the currently existing pion data are not
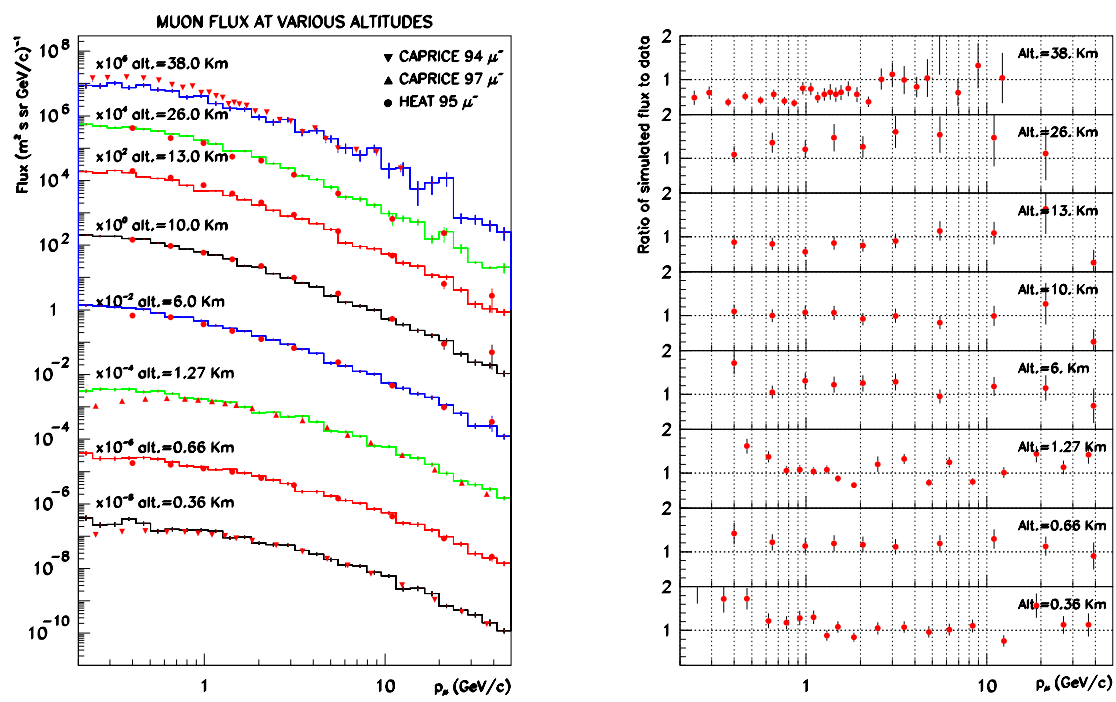

Fig. 5. Left: Experimental (full circles) and $\mu^{-}$flux measured by the BESS and CAPRICE experiments compared with simulated values (histograms, preliminary results). Right: Ratio of the experimental to simulated values. 
enough constraining for the relevant cross section parameters to be fixed, and the low energy $\pi$ yield cannot be calculated with a good enough accuracy for the $\mu$ yield to be reproduced accurately. However, this inaccuracy has a limited influence on the calculated neutrino flux ${ }^{14}$.

\subsection{Atmospheric neutrinos}

After the $e^{ \pm}$and $\mu^{ \pm}$have been calculated and shown to reproduce the measured data with a good enough accuracy, the last stage of the approach is the calculation of the neutrino flux. The calculations have been performed to span the range of geomagnetic coordinates, and the four neutrino energy spectra as well as the zenith and azimuth distributions have been calculated as a function of the latitude and longitude coordinates ${ }^{14}$.In addition, the flux have been calculated at the locations of the existing underground experiments. They have been used to calculate the East-West asymmetries $A_{E W}$ for the $e$-like and $\mu$-like events in the same conditions as reported by the SuperK experiment ${ }^{26}$, i.e. same cuts on the energy range, and compared to the experimental values. In addition, in the calculations, the $e$ and $\mu$ production angles have been approximately taken into account by using a functional form adjusted on the values reported in ${ }^{26}$. The preliminary results for the mean values of $A_{E W}$ calculated in the above conditions for the two class of events are in quite good agreement with the superK measurements. The experiment reported: $A_{E W}^{e-l i k e}(\exp )=0.21 \pm 0.04$ and $A_{E W}^{\mu-l i k e}(\exp )=0.08 \pm 0.04$, to be compared to the values obtained in the simulation: $A_{E W}^{e-l i k e}(\operatorname{sim})=0.27 \pm 0.1$ and $A_{E W}^{\mu-l i k e}(\operatorname{sim})=0.092 \pm 0.05^{10,14}$.

\section{Summary and outlook}

In summary, it has been shown that the simulation model described in this presentation allows to successfully account for the charged particle flux measured by AMS01 below the geomagnetic cutoff at satellite altitudes, and for the hadron ( $p$, $\bar{p}$ ) and lepton $\left(e^{ \pm}, \mu^{ \pm}\right)$flux measured in the atmosphere down to ground level, as well as in the underground superK neutrino experiment. Final calculations are currently being processed for atmospheric antiprotons and leptons.

Finally, the numerical tool developed for these analysis should also provide a powerful mean of estimating the atmospheric flux of secondary particles for future satellite experiments like AMS02, PAMELA, GLAST, etc..., as well as for balloon and underground (like SOUDAN) experiments. It is also clearly applicable to the investigation of other dynamical ranges of CR-atmosphere interactions, such as the mechanism and the dynamics of population of the radiation belts in a much lower energy domain, and likely to other similar topics of geophysical interest.

\section{Acknowledgements}

The author is indebted to his collaborators, L. Derome, Yong Liu, B. Baret, and C.Y. Huang, for their help in the preparation of this talk. 


\section{References}

1. AMS Collaboration, J. Alcaraz et al., Phys. Lett. B 472, 215(2000).

2. AMS Collaboration, J. Alcaraz et al., Phys. Lett.B 490, 27(2000)

3. AMS Collaboration, J. Alcaraz et al., Phys. Lett. B 484, 10(2000).

4. AMS Collaboration, J. Alcaraz et al., Phys. Lett.B 494, 193(2000).

5. L. Derome et al., Phys. Lett. B 489, 1(2000); L. Derome, and M. Buénerd, Nucl. Phys. A 688, 66c (2001).

6. L. Derome, M. Buénerd, and Yong Liu, Phys. Lett. B 515, 1(2001).

7. L. Derome, and M. Buénerd, Phys. Lett. B 521, 139(2001).

8. L. Derome and M. Buénerd, 27th ICRC, Hamburg, Aug. 7-14, 2001, OG1.019-20.

9. L. Derome, Yong Liu, and M. Buénerd, ref [8], OG1.018

10. Yong Liu, L. Derome, and M. Buénerd, ref [8], OG2.02

11. C.Y. Huang, L. Derome, and M. Buénerd, ref [8], OG1.014

12. C.Y. Huang and M. Buénerd, Parametrization of the inclusive antiproton cross section produced by 11 to 24 GeV protons on nuclei, Report No. ISN-01/18, March 2001 (unpublished).

13. C.Y. Huang, M. Buénerd, and L. Derome, in preparation; C.Y. Huang, PhD thesis, Université J. Fourier, Grenoble, in preparation.

14. Yong Liu, L. Derome, and M. Buénerd, Atmospheric muon and neutrino flux from 3-dimensional simulations, In preparation.

15. L. Derome, private communication.

16. A. N. Kalinovsky, N. V. Mokhov, and Y. P. Nikitin, "Passage of High Energy Particles Through Matter", New-York, AIP ed., 1989.

17. Yong Liu, L. Derome, and M. Buénerd, Parametrization of the inclusive production cross section of proton induced $\pi^{ \pm}$particles on nuclei, ISN Internal Report 01-012, Feb 2001 (unpublished).

18. Y.D. Bayukov et al., Sov. J. Nuc. Phys. 42, 116(1985).

19. T. Maeno et al., AstroPart. Phys. 16, 121(2001)

20. M. Fujikawa, PhD thesis, University of Tokyo, 2001; M. Nozaki, private communication; and BESS collaboration, to be published.

21. M. Boezio et al., ApJ 561, 787(2001)

22. A. Menchaca-Rocha et al., Private communication.

23. J. Kremer et al., Phys. Rev. Lett. 83, 4241(1999)

24. S. Coutu et al., Phys. Rev. D 62, 032001(2000)

25. T. Kajita and Y. Titskuba, Rev. Mod. Phys.73, 85(2001)

26. Y. Fukuda et al., Phys. Rev. Lett. 81, 1562(1998)

27. T. Fugutami et al., Phys. Rev. Lett. 82, 5194(1999) 